\title{
CAPÍTULO 39: EFICIÊNCIA NO USO DE ÁGUA EM PRODUTORES DE MORANGO NA LOCALIDADE DE SÃO PEDRO, MELIPILA, REGIÃO METROPOLITANA, CHILE
}

\section{CAPÍTULO 39: EFICIENCIA EN EL USO DE AGUA EN PRODUCTORES DE FRUTILLA DE LA LOCALIDAD DE SAN PEDRO, MELIPILLA, REGIÓN METROPOLITANA, CHILE}

\section{CHAPTER 39: EFFICIENCY IN THE USE OF WATER IN STRAWBERRY PRODUCERS IN THE LOCALITY OF SAN PEDRO, MELIPILLA, METROPOLITAN REGION, CHILE}

Andrea Pilar Baeza Villarroel ${ }^{1}$; Natalia Ovalle²; Néstor Salas ${ }^{3}$; Ronald Sánchez ${ }^{4}$; Sebastian Martínez ${ }^{5}$

DOI: https://doi.org/10.31692/978-65-88970-07-2.348-352

\section{INTRODUCCIÓN}

La producción de frutillas en Chile se concentra principalmente en las regiones del Maule y Metropolitana, las cuales abarcan el 80\% de la superficie cultivada en el país (Pefaur Lepe, 2014). En San Pedro, comuna ubicada en la Provincia de Melipilla, Región Metropolitana, se encuentra el principal nicho productivo de frutillas para mercados internacionales y nacionales de la zona central del país (Municipalidad de San Pedro, 2019), principalmente en manos de pequeños agricultores que en promedio cuentan con 0,5 a 2 hectáreas productivas, lo que constituye la base del ingreso monetario para una gran cantidad de agricultores de la comuna (Consultorías Profesionales Agraria Ltda., 2015).

La comuna se caracteriza por tener un clima semiárido, con influencia costera, la información climatológica de la zona en los últimos 30 años, nos indica que la temperatura media es de 13,9 ${ }^{\circ} \mathrm{C}$, con una humedad relativa de $79 \%$, precipitaciones anuales de $396 \mathrm{~mm}$, evapotranspiración potencial de $1230 \mathrm{~mm}$ y un déficit hídrico acumulado de $987 \mathrm{~mm}$, evidenciando los efectos del cambio climático y aumento en la escases del recurso hídrico de la zona (Santibañez, y otros, 2014). La provincia de Melipilla ha sido declarada "zona de escasez hídrica" durante varios años consecutivos, indicando la fuerte falta del recurso y la sequía que afecta a la zona en los últimos 10 años, es por esta razón que es vital realizar un manejo eficiente del recurso hídrico, implementado técnicas de eficiencia de uso de agua y

\footnotetext{
1 Ingeniera agrónoma, Docente INACAP, andrea.baeza03@inacapmail.cl

2 Estudiante Ingeniería Agrícola INACAP, natalia.ovalle02@inacapmail.cl

3 Estudiante Ingeniería Agrícola INACAP, nestor.salas@,inacapmail.cl

${ }^{4}$ Estudiante Tecnología Agrícola INACAP, ronald.sanchez02@inacapmail.cl

5 Estudiante Ingeniería Agrícola INACAP, sebastian.martinez58@inacapmail.cl
} 
teniendo en consideración que más del 70\% del reservorio de agua del país es destinado al rubro agropecuario.

\section{INFORME DE EXPERIENCIA}

Metodología: El objetivo de esta experiencia fue determinar la eficiencia del uso de agua en los productores de frutilla de la comuna de San Pedro, Provincia de Melipilla, Región Metropolitana, de tal forma de fortalecer el ámbito productivo de la zona. Para poder cuantificar el uso de agua en los predios de frutillas, se elaboró una encuesta y lista de verificación, que abarcó los principales usos de agua directos dentro de la producción primaria de frutillas. La encuesta abarcó los ítems de mayor relevancia, como: variedades utilizadas superficie de cultivo, características de suelo, tipo de riego, uso de agua de riego y de otras labores agrícolas, entre otras. Las encuestas fueron realizadas entre mayo y septiembre del año 2019. Se aplicaron 4 encuestas a agricultores que trabajan coordinadamente con la empresa Agrofrutillas San Pedro S.A., que agrupa más de 30 productores de frutilla de la zona.

Caracterización de las zonas productivas: Los productores corresponden principalmente a pequeños agricultores, con menos de 5 hectáreas cultivadas. Dentro del universo de agricultores entrevistados el promedio de superficie con frutillas fue de 1,93 hectáreas y las variedades utilizadas por los encuestados son Monterey, Cabrillo y Portola (Tabla 1). Las variedades utilizadas son de día neutro, es decir, que no requieren de fotoperiodo para fructificar, proceso que sucede cuando las temperaturas están por sobre los $12^{\circ} \mathrm{C}$. Estas variedades se pueden plantar en distintos meses del año, como en verano (enero febrero), inicios de invierno (mayo) y primavera (septiembre - octubre), la fecha de plantación determinará la etapa productiva de las variedades, abarcando desde septiembre hasta abril (Undurraga \& Vargas, 2013). 
Tabla 1. Características generales de los predios encuestados en San Pedro, Provincia de Melipilla, Región Metropolitana, Chile.

\begin{tabular}{lrrrr}
\hline \multirow{2}{*}{ Características del cultivo } & \multicolumn{4}{c}{ Predio } \\
\cline { 2 - 5 } Superficie cultivada (ha) & $\mathbf{2}$ & $\mathbf{2}$ & $\mathbf{3}$ & $\mathbf{4}$ \\
Variedades & 1 & 2 & 4 & 0,72 \\
Fecha plantación & Monterey & Monterey & Portola / Cabrillo & Portola / Cabrillo \\
Rendimiento (ton/ha) & Marzo 2019 & Octubre 2018 & Junio 2019 & Junio 2019 \\
\hline \multicolumn{2}{c}{ Fuente. Elaboración propia (2019). } & 84 & 60 \\
\hline
\end{tabular}

El suelo es uno de los recursos clave para la productividad y manejo del agua en el cultivo de frutilla. La textura del suelo es un componente físico que permite retener o almacenar agua en el suelo para ser utilizada por los cultivos. Los agricultores encuestados presentan suelos del tipo franco arcilloso a arcillo arenoso. Los cuales presentan un contenido entre 20 a $65 \%$ de arena, 15 a $50 \%$ de limo y entre 27 a 55\% de arcilla, lo que los hace suelos con una buena retención de agua, porosidad y drenaje, adecuados para frutillas (Ferreyra \& Sellés, s.a.). La profundidad de suelo en los predios encuestados variaba entre 100 y $120 \mathrm{~cm}$, mientras que la profundidad de raíces puede llegar hasta los $50 \mathrm{~cm}$ en cultivos en plena producción.

Huella hídrica: Dentro de las actividades que utilizan agua en la producción primaria de frutillas, se evaluó su uso en agua de riego, limpieza de implementos de cosecha, aplicación de productos fitosanitarios y finalmente uso en servicios higiénicos y agua de bebida del personal de trabajo de cada predio. En la Tabla 2 se observan los valores obtenidos para determinar la eficiencia en el uso de agua, en $\mathrm{m}^{3}$ de agua utilizada por tonelada de fruta producida.

Tabla 2. Uso de agua directa en $\mathrm{m}^{3}$ por temporada y eficiencia en el uso de agua (EUA) en 4 predios encuestados de San Pedro, Provincia de Melipilla, Región Metropolitana, Chile.

\begin{tabular}{|c|c|c|c|c|c|c|}
\hline \multirow[b]{2}{*}{ Ítems } & \multicolumn{6}{|c|}{ Uso de agua $\left(\mathrm{m}^{3} /\right.$ temporada) } \\
\hline & $\begin{array}{c}\text { Predio } \\
1\end{array}$ & $\begin{array}{l}\text { Predio } \\
2\end{array}$ & Predio 3 & Predio 4 & $\begin{array}{c}\text { Promedi } \\
\text { o }\end{array}$ & Participación \\
\hline Servicios higiénicos & 15,0 & 20,0 & 17,0 & ND & 17,3 & $0,27 \%$ \\
\hline Agua bebida & 2,0 & 1,0 & 0,5 & ND & 1,2 & $0,02 \%$ \\
\hline Lavado bandejas & 4,0 & 2,5 & 2,0 & 8,4 & 4,2 & $0,06 \%$ \\
\hline Aplicaciones Agroquímicos & 3,6 & 12,0 & 6,0 & 11,2 & 7,2 & $0,11 \%$ \\
\hline Riego & $\begin{array}{r}16.933, \\
3\end{array}$ & $\begin{array}{r}12.692, \\
3\end{array}$ & $\begin{array}{r}6.095,2 \\
2 \\
\end{array}$ & ND & $11.907,0$ & \\
\hline Total (m³/Ha/temporada) & $\begin{array}{r}16.957, \\
9 \\
\end{array}$ & $\begin{array}{r}12.727, \\
8 \\
\end{array}$ & $6.120,7$ & ND & $11.936,9$ & 00,0 \\
\hline Producción temporada (Ton/Ha) & 20,0 & 80,0 & 84,0 & 60 & 61,0 & \\
\hline EUA $\left(m^{3} /\right.$ Ton fruta $)$ & 847,9 & 159,1 & 72,9 & ND & 195,7 & \\
\hline
\end{tabular}

Fuente: Elaboración propia (2019).

La huella hídrica o agua consumida que pasa a formar parte del producto ha sido 
estudiada en numerosas investigaciones. Estudios realizados en Córdoba Argentina, determinan que se requieren 106,1 $\mathrm{m} 3$ de agua para producir una tonelada de frutillas, bajo las condiciones de mulch plástico y microtúneles (Domínguez \& Chauvet, s.a.). Mientras que ensayos llevados a cabo en España durante la temporada 2014/15 demuestran que se necesitan $47 \mathrm{~m}^{3}$ de agua por tonelada de fruta producida (Aguilar \& Orlando, 2018). Existe gran diferencia entre distintos estudios realizados a nivel mundial, principalmente debido a que en el uso de agua influyen fuertemente las condiciones edafoclimáticas de la zona y los manejos agronómicos del cultivo, es así como cada país o sistema productivo local debiese calcular y manejar sus propios valores de uso de agua, para generar las medidas de mitigación. Esta misma variabilidad se puede observar en los datos recolectados en San Pedro, en donde los valores de uso de agua van desde los 73 a $\operatorname{los} 857 \mathrm{~m}^{3}$ de agua por tonelada de frutilla producida, con un promedio de $196 \mathrm{~m}^{3} /$ ton, valores superiores a los consultados en la literatura. Las principales pérdidas de agua encontradas fueron correspondientes al riego, el cual no cuenta con una programación calculada específicamente para la zona y cultivo. Las diferencias entre cada agricultor encuestado fueron importantes y se debe proponer una alternativa para disminuir esta brecha.

\section{CONCLUSIONES}

La comuna de San Pedro, ubicada en la Provincia de Melipilla, es la principal zona productiva de frutillas de la Región Metropolitana, destinando su producción directamente al mercado fresco y agroindustrial nacional e internacional. La localidad ha sido declarada con escasez hídrica, presentado cerca de 10 años continuos de sequía, con un déficit hídrico promedio de 1000 milímetros. El uso eficiente de agua se hace primordial, para poder mantener el sustento productivo de la comuna, que está principalmente en manos de pequeños agricultores. Los cuales a lo largo de los últimos años han disminuido la superficie cultivada, debido a la sequía. Dentro del uso de agua directo en la producción primaria de frutillas, un $99,75 \%$ corresponde al agua utilizada para riego, siendo el principal componente que marca la eficiencia del sistema productivo. En general, el sector no cuenta con tecnologías y cálculos agronómicos para la programación del riego, haciendo un uso excesivo del recurso, en una zona de escasez hídrica. Se hace imperativo aplicar tecnologías, programación predictiva y control de los métodos de riego, acompañado con capacitaciones directas a los agricultores. 


\section{REFERENCIAS}

AGUILAR, T., \& ORLANDO, D. Utilidades y limitaciones del indicador Huella de agua de riego aplicado al cultivo de fresa en el entorno del Parque Nacional Doñana. Universidad de Cordoba, España. 2018.

CONSULTORÍAS PROFESIONALES AGRARIA LTDA. Diagnóstico Planes Marcos de Desarrollo Territorial (PMDT): Territorio 1 San Pedro. Santiago, Chile: Gobierno de Chile. 2015.

DOMÍNGUEZ, V., \& CHAUVET, S. (s.a.). Huella de agua: un punto de partida pra la reducción de agua en un empaque de frutillas. Facultad de Ciencias Exactas y Tecnología, Universidad Nacional de Tucumán, Argentina.

FERREYRA, R., \& SELLÉS, G. Manual de riego para especies frutales: uso eficiente del agua de riego y estrategias para enfrentar períodos de escasez. Santiago, Chile: Boletín INIA $\mathrm{N}^{\circ} 278.2013$.

MORALES, C. Manual de manejo agronómico de la frutilla. 17. Municipalidad de San Pedro. (Diciembre de 2019). https://www.munisanpedro.cl. Obtenido de https://www.munisanpedro.cl/comuna/\#1514403822675-f982e675-d631. 2017.

SANTIBAÑEZ, F., SANTIBAÑEZ, P., CAROCA, C., MORALES, P., GONZÁLEZ, P., GAJARDO, N., MELILLÁN, C. Atlas del cambio climático en las zonas de régimen árido y semiárido: Regiones de Coquimbo, Valparaíso y Metropolitana, Chile. Santiago: Centro AGRIMED, Facultad de Ciencias Agronómicas, Universidad de Chile. 2014.

UNDURRAGA, P., \& VARGAS, S. (2013). Manual de Frutilla. Boletín INIA, 262. 